\title{
Use of a Double Resonance Electron Capture Dissociation Experiment to Probe Fragment Intermediate Lifetimes
}

\author{
Cheng Lin \\ Mass Spectrometry Resource, Department of Biochemistry, Boston University School of Medicine, Boston, \\ Massachusetts, USA
}

Jason J. Cournoyer

Department of Chemistry, Boston University, Boston, Massachusetts, USA

\section{Peter B. O'Connor}

Mass Spectrometry Resource, Department of Biochemistry, Boston University School of Medicine, Boston, Massachusetts, USA

\begin{abstract}
The relative abundances of fragment ions in electron capture dissociation (ECD) are often greatly affected by the secondary and tertiary structures of the precursor ion, and have been used to derive the gas-phase conformations of the protein ions. In this study, it is found that resonance ejection of the charge reduced molecular ion during ECD resulted in significant changes in many fragment ion populations. The ratio of the ion peak intensities in the double resonance (DR)-ECD to that in the normal ECD experiment can be used to calculate the lifetime of the radical intermediates from which these fragments are derived. These lifetimes are often in the ms range, a time sufficiently long to facilitate multiple free radical rearrangements. These ratios correlate with the intramolecular noncovalent interactions in the precursor ion, and can be used to deduce information about the gas-phase conformation of peptide ions. DR-ECD experiments can also provide valuable information on ECD mechanisms, such as the importance of secondary electron capture and the origin of c./z ions. (J Am Soc Mass Spectrom 2006, 17, 1605-1615) (c) 2006 American Society for Mass Spectrometry
\end{abstract}

$\mathrm{F}$ or peptides and proteins, electron capture dissociation (ECD) [1-5] cleaves more evenly along the backbone than conventional MS/MS methods (e.g., collisionally activated dissociation, CAD, and infrared multiphoton dissociation, IRMPD) while preserving the more labile post-translational modifications (PTMs), making it a very useful tool in the field of proteomics [1-4, 6-10]. Such behavior has been suggested to be evidence of a nonergodic dissociation pathway, in which much of the $\sim 6 \mathrm{eV}$ neutralization energy was utilized locally before randomization, allowing competitive cleavages of backbone bonds with significantly different dissociation energies, and preservation of more labile side-chain bonds, even noncovalent interactions. However, there is also a body of calculations that suggest that electron capture produces a long-lived aminoketyl radical species for which backbone cleavage is the lowest energy pathway, therefore eliminating the need to evoke the nonergodicity assumption [5, 11, 12].

In addition to a rapid, possibly nonergodic primary cleavage, ECD generates a metastable radical cation as

Published online August 9, 2006

Address reprint requests to Professor P. B. O'Connor, Mass Spectrometry Resource, Department of Biochemistry, Boston University School of Medicine, 670 Albany St., Boston, MA 02118, USA. E-mail: poconnor@bu.edu one of the product species [11, 13]. ECD of cyclic peptides showed extensive secondary side-chain and backbone cleavages, which was postulated to be the result of a free radical cascade (FRC) [5]. The FRC postulated that the $\alpha$-radical produced from the initial electron capture migrated along the backbone to initiate multiple free radical rearrangements, causing multiple backbone cleavages and additional side-chain cleavages. Radical rearrangement was also observed in ECD of a linear peptide, where an isotopic pattern shift in the deuterium substituted peptide was the result of $\mathrm{H} / \mathrm{D}$ scrambling [12]. Although unable to prove the free radical cascade, as the scrambling could also result from the intermolecular hydrogen transfer after the backbone cleavage, this experiment clearly demonstrated the presence of a long-lived radical intermediate in ECD.

The presence of a reaction intermediate can sometimes be revealed via double resonance (DR) experiments [14]. In essence, on-resonant ejection of a specific ion during a dissociation event should modulate the abundance of all secondary ions derived from it. Originally developed to identify chemically coupled ionmolecule reactions in complex mixtures in ion cyclotron resonance (ICR) studies [15], DR was later adapted to Fourier-transform mass spectrometry (FT-MS) [16, 17], and other kinds of mass spectrometry [18], and was 
used to study the mechanisms of CAD and blackbody infrared dissociation (BIRD) [18-20]. In addition to revealing the connection between two observed species, the DR method can also be applied to determine the existence of transient intermediates, as demonstrated by Glish and coworkers in the collisionally induced dissociation (CID) of $[\mathrm{YG} \beta \mathrm{FL}+\mathrm{H}]^{+}$ion in a quadrupole ion trap [14]. Furthermore, with DR, it is also possible to measure the lifetime of reaction intermediates if they fall within the range of ejection times, typically from tens of $\mu$ s to several ms. Using DR, Anicich et al. were able to determine the mean lifetime of the collision complex $\mathrm{H}_{2} \mathrm{C}_{6} \mathrm{~N}_{2}^{+}$in the ion-molecule reaction between $\mathrm{HC}_{3} \mathrm{~N}^{+}$and $\mathrm{HC}_{3} \mathrm{~N}$ to be $180 \mu \mathrm{s}$ [21]. In one occasion, the DR method has been applied to ECD studies in Zubarev's group, although no significant changes in fragment ion distribution were observed upon resonance ejection of the charge reduced species, leading to the conclusion that secondary electron capture was insignificant under their conditions [22].

Over the last decade, extensive research efforts have been dedicated to the study of protein conformations in the gas phase, as its comparison to solution phase conformations would enable us to understand the effects of aqueous solvation. Among these studies are a variety of MS based measurements, such as H/D exchange [23-26], collision cross section (ion mobility MS) [27-30], photofragment spectroscopy [31, 32], and ECD [31, 33-35]. Because ECD preferentially cleaves protein backbone bonds without breaking tertiary noncovalent bonds, the ECD fragment ion abundances are greatly affected by the secondary and tertiary structures of gaseous protein ions. Thermal denaturation and IR photon absorption often weaken these noncovalent interactions, leading to diminished effect on the ECD fragment ion intensities. Capitalizing on this, McLafferty and coworkers were able to postulate conformational structures for gaseous protein ions based on their ECD spectra, and to study their gas-phase folding kinetics [31, 33, 34]. Although most of such studies are done on protein ions, small peptide ions can also have specific gas-phase conformations that may affect the observed ECD fragment ion abundance distributions. ECD of substance $P$ and cyclic peptide gramicidin $S$ in a cryogenic ICR cell $(86 \mathrm{~K})$ produced far less fragment ions than when done at room temperature, indicating reduced conformational heterogeneity at low temperatures [36].

In this paper, DR-ECD studies on several peptide ions are presented. The spectra show that the onresonant ejection of the charge reduced species often greatly alters the ECD fragment ion distribution, which can be used as a sensitive probe for gas-phase ion conformation studies. Additionally, these experiments show that many ECD fragments are formed via a long-lived radical intermediate, whose lifetime can often be measured in a DR-ECD experiment.

\section{Materials and Methods}

Experiments were carried out on a previously described hybrid Fourier transform ion cyclotron resonance (FTICR) mass spectrometer with an electrospray ionization (ESI) source [37, 38]. All peptides were purchased from Sigma (St. Louis, MO), except for BUSM 1, which was synthesized by Anaspec (San Jose, CA), and BUSM 1a, which was synthesized by performing the tryptic digest of peptide BUSM 1 to remove the $N$-terminal arginine. The samples were diluted to $\sim 1$ to $5 \mathrm{pmol} / \mu \mathrm{L}$ in 50:50:1 $\mathrm{H}_{2} \mathrm{O}: \mathrm{CH}_{3} \mathrm{OH}: \mathrm{HCOOH}$ solution, and electrosprayed from pulled glass capillary nanospray tips to generate multiply charged ions. An indirectly heated dispenser cathode (model STD200, Heatwave, Watsonville, CA) was heated with $4 \mathrm{~V}$ and $0.8 \mathrm{~A}$ to generate the low-energy electrons needed for ECD. It was mounted on a separate stainless steel support $\sim 10 \mathrm{~cm}$ away from the ICR cell, and the cell trap plates were all polished to a mirror shine to minimize radiative heating of trapped ions. Electron energy can be adjusted by varying the body potential of the dispenser cathode. In a typical ECD experiment, the trapped ions were subjected to 2 to $10 \mathrm{~ms}$ irradiation of $\sim 0.5 \mathrm{eV}$ electrons. In double resonance experiments, the charge reduced species were ejected from the cell by applying a single frequency excitation with a peak to peak amplitude of 5 to $40 \mathrm{~V}$ during the electron irradiation period.

The spectra were acquired with a Nyquist frequency corresponding to a low $m / z$ limit of $\sim 215$, zero filled twice, and Fourier transformed without apodization. In normal ECD experiments, internal calibration on the $[\mathrm{M}+$ $\mathrm{nH}]^{\mathrm{n}+}$ and $[\mathrm{M}+\mathrm{nH}]^{(\mathrm{n}-1)+\cdot}$ and their isotopic peaks yielded a typical mass accuracy of 1 to $2 \mathrm{ppm}$. The same calibration file was then used in the corresponding DR-ECD spectrum, yielding a typical mass accuracy of $<5 \mathrm{ppm}$.

\section{Results and Discussion}

\section{DR-ECD of a Linear Peptide (Fibrinopeptide B)}

ECD of fibrinopeptide B (EGVNDNEEGFFSAR) generated a complete $\mathrm{z}$-ion series down to $\mathrm{m} / \mathrm{z} \sim 215$ corresponding to the Nyquist frequency limit used (Figure 1a). ECD fragments are often considered as a snapshot of the ion conformations at the time of electron capture dissociation, with possible tertiary structures hindering the separation of product ions. It is tempting to claim that this peptide molecular ion has negligible tertiary structures, as all inter-residue cleavages were readily observed. However, the current ECD "camera" has one major drawback in its slow shutter speed. An ECD experiment often involves a delay of at least hundreds of $\mathrm{ms}$ (and often several seconds) between the dissociation event (shutter open) and the product ion excitation/detection (shutter close and film development). During this period, the noncovalent bonds holding the fragment ion pair together could well break down, possibly due to the excess energy deposited by electron capture and the Columbic repulsion of the fragment 


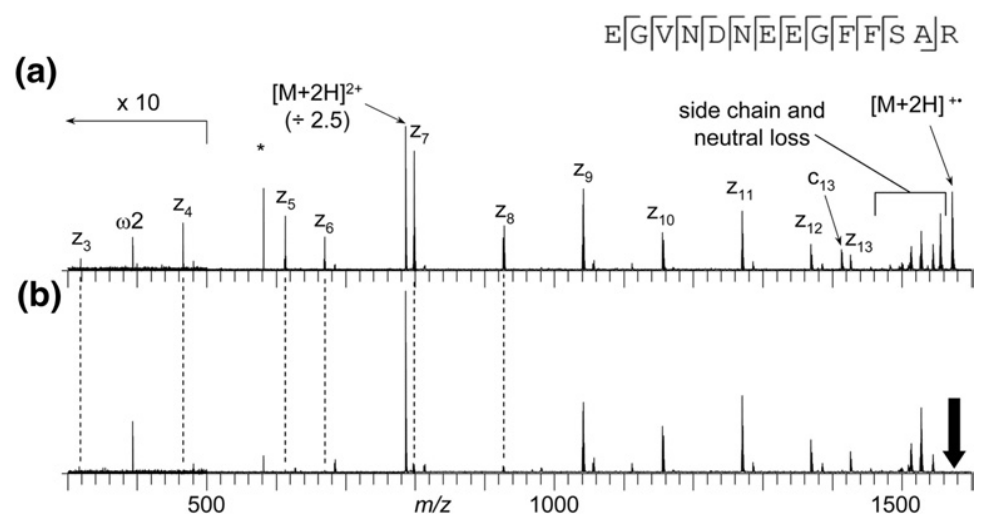

Figure 1. ECD spectra of fibrinopeptide B without (a) and with (b) resonant ejection of the charge reduced molecular ion.

ions, or simply due to the slow heating by collisions with background gas molecules and background blackbody radiation, thus taking away conformation information typically stored in the missing cleavages. Sometimes, the lost information can be restored by observing fragment ion abundance increase upon ion activation via heating or IR irradiation, [33, 34] but this approach does not always work: if all pixels are bright (as in the case when all fragment ions were separated before detection), overexposure (ion activation) will not lead to image sharpening. The image "smearing" problem is particularly severe in the case of small peptide ions, where noncovalent interactions are often weaker than those of larger protein ions. One would have to develop a faster shutter that allows image capturing at the time of dissociation to observe these fleeting interactions.

Since ECD is initiated by capture of an electron to generate the charge reduced species, the $[\mathrm{M}+2 \mathrm{H}]^{+}$. ion, through which all fragment ions are formed, a DR experiment with excitation at that $\mathrm{m} / \mathrm{z}$ (Figure 1b, marked with an arrow) should provide a rough measurement of the product ion lifetimes for all fragments, which should correlate with the strength of various noncovalent interactions. Figure $1 \mathrm{~b}$ shows the ECD spectrum of fibrinopeptide B upon resonant ejection of the charge reduced molecular ion $\left(m / z \sim 1571.7, \mathrm{~V}_{p-p}=\right.$ $40 \mathrm{~V})$. The peak intensity of every $z$-ion smaller than $z_{9}$ decreased sharply, but that of $z_{9}$ and larger ions remained roughly the same. Previous studies have shown that, after the initial $\mathrm{N}-\mathrm{C} \alpha$ bond cleavage, the $\mathrm{c} / \mathrm{z}$ ion pair generated by ECD may still be held together by noncovalent interactions such as hydrogen bonds and salt bridges, preventing the detection of individual fragments [33]. Fibrinopeptide B has an acidic residue rich region, and the side-chains of these residues could form multiple hydrogen bonds with the C-terminal arginine. When ECD cleaved any bond in or to the right (C-terminal) side of this region, the resulting $c(n e u-$ tral)/z. ion pair would still be held together by these hydrogen bonds, and might have a lifetime too long to dissociate before they were ejected from the cell. It is thus of no surprise that small $\mathrm{z}$ fragments showed steeper drop in abundance as the complementary $c$ neutrals have more acidic residues available to interact with the terminal arginine, either as neutral hydrogen bonds or as ionic interactions. $z_{9}$ and $z_{10}$ ions showed only slight decreases in their peak intensities despite the presence of an Asn and Asp residue, respectively, on their complementary $\mathrm{c}$ neutrals. This is due to the conformation of the peptide ion that produced them. As all $\mathrm{z}$ ions retained charge at the $\mathrm{C}$-terminal arginine side-chain, the neutralized charge had to come from the $\mathrm{N}$-terminal $-\mathrm{NH}_{3}^{+}$group. The classic picture of ECD has the $\mathrm{N}-\mathrm{C} \alpha$ bond cleavage occurring C-terminal to the charge solvated carbonyl, thus the $\mathrm{z}_{9 / 10}$ generating peptide ion should have the $\mathrm{N}$-terminus hydrogen bonded to either Asn4 or Asp5, preventing Arg14 from binding there.

\section{Lifetime of Radical Intermediates}

As previously shown, the lifetime of reaction intermediates can be measured using DR, provided the lifetime is within the resonance ejection timeframe. Figure $2 \mathrm{a}$ shows part of the ECD spectra of fibrinopeptide B with various ejection voltages. The ejection time $t$ relates to the excitation voltage (peak-to-peak) $\mathrm{V}_{p-p}$ via eq 1 [21]

$$
t=2 d r B / V_{\mathrm{pp}}
$$

where $d$ is the cell diameter, $r$ is the ejection radius that can be approximated as $d / 2$, and $B$ is the magnetic field. The drop in fragment ion abundances as a function of $\mathrm{V}_{p-p}$ directly correlates with the number of ions ejected and, hence, the number of ions that survive for at least time $t$. Figure $2 \mathrm{~b}$ plots the abundance of "surviving" intermediates as a function of time for the $z_{7}$ and $z_{8}$ ions. Since fragmentation of the $\mathrm{H}$-bonded intermediate complex is a unimolecular dissociation, these values can be fit to a single exponential and the lifetimes were calculated to be 2.1 and $1.8 \mathrm{~ms}$, respectively. This is sufficiently long to allow multiple radical rearrangements, such as those observed in the cyclic peptide 
(a)

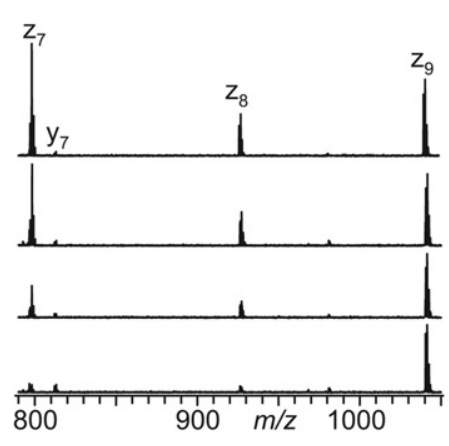

(b)

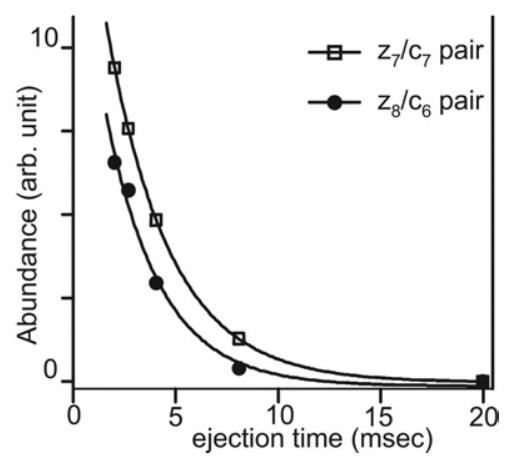

Figure 2. (a) Expanded region of ECD spectra of fibrinopeptide B with reduced molecular ion ejection at various ejection voltage; from top to bottom: 0, 10, 20, and $40 \mathrm{~V}$. (b) First-order decay of the remaining ion pair abundance as a function of ejection time (as calculated from ejection voltage according to eq 1). Solid lines are the single exponential fits.

experiment [5], and the H/D scrambling experiment [12].

It is important to point out that the above lifetime calculation assumes that all fragment ions produced before complete ejection of the corresponding intermediates could be detected with the same efficiency as those produced in the center of the cell. In reality, partial excitation of the ions would destroy the ion coherence for subsequent cyclotron orbit excitation, resulting in decreased detection efficiency. Strict treatment of this issue is beyond the scope of the current paper, but the net result is an approximately 2 -fold overestimate of the intermediate lifetimes. Once we realize that the upper limit of the lifetime of these intermediates is in the ms range, it is easy to understand why conventional ECD failed to reveal the existence of these noncovalent interactions in small peptides, as the delay between the ECD event and fragment ion detection is often two orders of magnitude longer than what it takes for fragment ions to separate. The DR-ECD experiment, on the other hand, "freezes" the ion conformation within a ms upon electron capture by ejecting slow-separating intermediates out of the cell long before the detection event. Using the camera analogy, DR-ECD captures the ion conformation image with a ms shutter in a film negative, which can later be developed. Last but not least, while the calculated lifetimes are only approximate in absolute terms, their relative values should still give good indication of the relative strengths of the various noncovalent interactions in the molecular ion.

\section{Fragment Ion Abundances in DR-ECD Reflect H-Bonds}

The presence of a hydrogen bond between $C$-terminal arginine and acidic residues in fibrinopeptide $B$ was suggested based on different behavior of $z_{8}$ and $z_{9}$ ions upon reduced ion ejection. The possibility of relating the fragment ion formation time (or the dissociation time of the corresponding intermediate) to the structure of the precursor ion is a reasonable hypothesis but required further testing. Such a test can be performed experimentally by demonstrating dissimilar behavior of a similar peptide with the acidic residues replaced by hydrophobic residues, or an isomeric analog with acidic residues at different positions.

To this end, we have carried out ECD and DR-ECD experiments on a pair of peptides: BUSM 1 (RAAAGADGDGAGADAR) and BUSM 1a (AAAGADGDGAGADAR). ECD of BUSM 1 produced $13 \mathrm{c}$ ions of relatively high abundance and seven less-abundant $\mathrm{z}$ ions (Figure 3a). This synthetic peptide has an arginine on each end, and three acidic residues spread out in the middle, giving rise to the possibility of forming multiple hydrogen bonds throughout the molecule. Because of this, most fragment ion peaks showed steep drop in intensity upon resonance ejection of the charge reduced species $(m / z \sim 1402.6$, Figure $3 b)$. However, four $c$ ions $\left(c_{6}, c_{8}, c_{13}\right.$, and $\left.c_{15}\right)$, showed only moderate changes in their abundances, and all but one $\left(c_{15}\right)$ of them resulted from cleavages $N$-terminal to an aspartyl residue. As the formation of all $c$ ions required the charge on the C-terminal arginine side-chain to be neutralized while it was hydrogen bonded to the carbonyl $\mathrm{N}$-terminal to the cleavage site, and the cleavage inevitably broke this hydrogen bond, the only possible noncovalent interaction that can hold the two fragment pieces together would be between the $N$-terminal arginine and one or more of the three aspartyl residues. The hydrogen bond between Arg16 and the carbonyl $\mathrm{N}$-terminal to an aspartyl residue necessary to generate cleavages is likely to disrupt some of these interactions, thus facilitating the separation of these fragment ions once they are formed.

Lack of $N$-terminal arginine in BUSM 1a peptide led to a very different ECD spectrum (Figure 3c) from that of BUSM 1. The predominant fragment ions were of the $\mathrm{z}$ type, indicating that the protonated $\mathrm{N}$-terminal amine was the preferential site of charge neutralization. Be- 

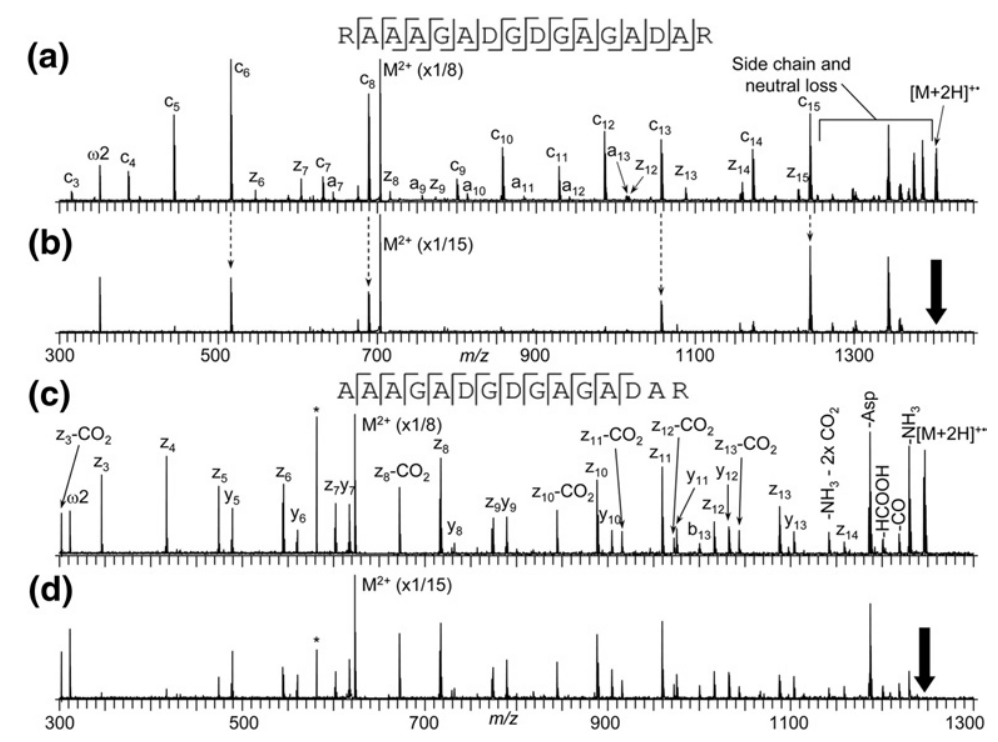

Figure 3. ECD (a) and DR-ECD (b) spectra of the BUSM 1 peptide, and the BUSM 1a peptide (c) and (d), respectively.

cause no $N$-terminal arginine was available to form multiple hydrogen bonds with the sidechains of aspartyl residues, the unique pattern of fragment ion abundance change for cleavages $N$-terminal to an aspartyl residue was not observed in its DR-ECD spectrum (Figure 3d). Instead, the DR-ECD spectrum displayed a trend similar to that observed in the fibrinopeptide $B$ experiment, namely, larger $\mathrm{z}$ ions showed little abundance change, while the smaller ones showed drastic drop in their abundances upon resonance ejection. The transition took place at $z_{8}$, once again, at a cleavage site one residue remote (to the $C$-terminal side) from the last available acidic residue (Asp6) that is capable of forming a hydrogen bond with the $\mathrm{C}$-terminal arginine (Arg15). The necessity of the $\mathrm{N}$-terminal $-\mathrm{NH}_{3}^{+}$group hydrogen bonding to the carbonyl of Gly7 to initiate the formation of $\mathrm{z}_{8}$ ion presented a steric hindrance for Arg15 to bind the nearby Asp6, agreeing with the hypothesis set forth in the fibrinopeptide B example.

The BUSM1 peptide is known for generating unusually high abundance of odd electron c. ions. A closer look at each individual $\mathrm{c}$ ion peak revealed that nearly every one of them showed steeper drop in its odd electron form than in its even electron form (Figure 4a), which is more apparent when the survival ratios (the ratio of the ion abundance with the application of on resonance ejection waveform to that without ion ejection) were plotted for each individual $c$ and $c$. ion (Figure $4 \mathrm{~b}$ ). Note that the Ionspec FTMS software (Irvine, CA) does not correct for ion charge when reporting ion abundance values, even though an ICR cell has a linear response to the number of charges. (a)

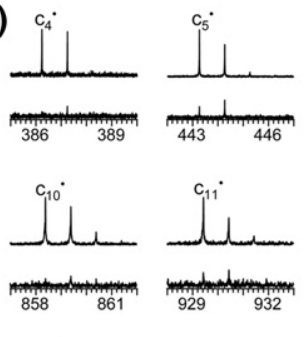

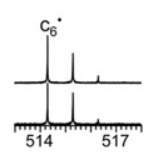
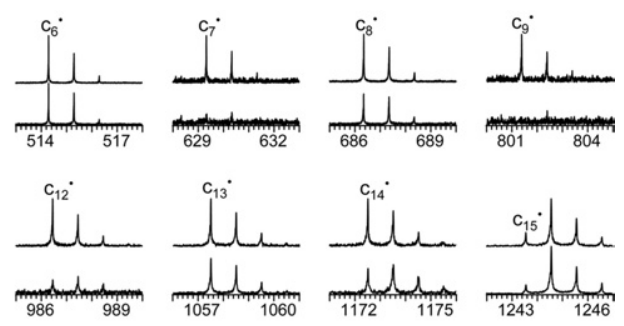

(b)

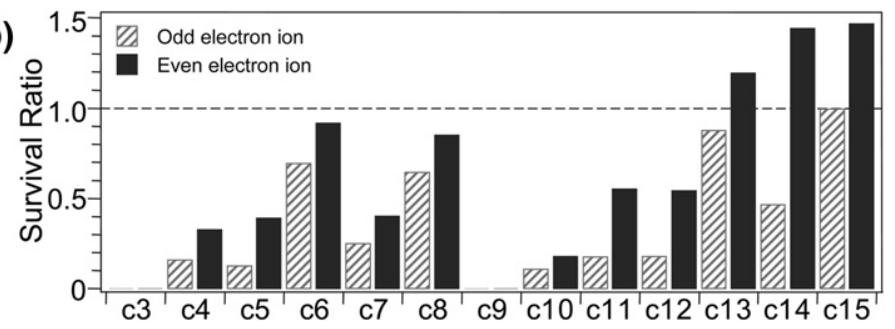

Figure 4. (a) Expanded regions of the ECD (top) and DR-ECD (bottom) spectra of the BUSM 1 peptide (from Figure 3(a) and (b)); (b) the ratio of ion abundance in DR-ECD spectrum versus that in the normal ECD spectrum (named "survival ratio") for all c-ions observed. 

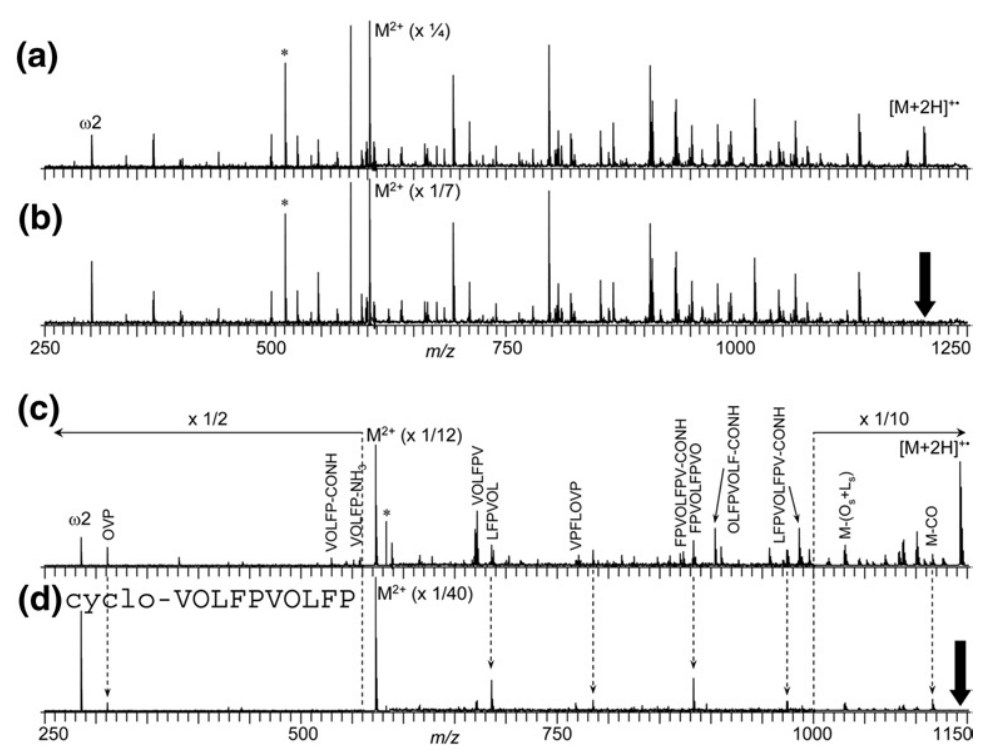

Figure 5. ECD and DR-ECD spectra of cyclosporin A (a) and (b), respectively, peaks labeled in reference [5], and gramicidin $\mathrm{S}(\mathbf{c})$ and (d), respectively.

However, in calculating the survival ratio, it is not necessary to correct for the charges as the ion abundance ratio is always taken for the same ion and, naturally, of the same charge state. Currently, it is believed that the initial ECD cleavage generates a $\mathrm{c} / \mathrm{z}$. ion pair, and the formation of $\mathrm{c}$ - ions comes from intermolecular hydrogen transfer, which can take up to ms to occur, thus making its population more susceptible to resonance ejection of the intermediates.

\section{DR-ECD of Cyclic Peptides}

All previous examples involve some kind of noncovalent interactions between two fragment pieces preventing their separation leading to their abundance drop in DR-ECD experiment. Intuitively, the intermediate with stronger interaction should have a longer lifetime, which in turn should result in a steeper drop in the corresponding individual fragment ion abundance upon its resonance ejection, as observed in the fibrinopeptide $B c_{7}$ to $c_{8}$ ions. Extending this argument to the case of cyclic peptide would lead to the conclusion that nearly all secondary backbone fragment ions should disappear in DR-ECD experiment, since the $\mathrm{c}$ and $\mathrm{z}$ parts produced by the initial cleavage were essentially just one piece, or "two pieces" held together by a covalent bond. However, this cannot be further from the truth as evident in the ECD and DR-ECD spectra of the cyclic peptide cyclosporin A (Figure 5a, structure shown in Scheme 1), where, much to our surprise, not a single fragment ion peak showed appreciable drop in its intensity upon charge-reduced molecular ion ejection. The only logical explanation to this is that if the free radical cascade was responsible for the generation of these secondary fragments, it must take place on a submillisecond timescale. In retrospect, this is perhaps not so surprising, as radical rearrangements often take place on a $\mu$ s timescale or faster [39]. In addition, cyclosporin $\mathrm{A}$ is also different from all previous peptides in that it contains no polar side-chains capable of forming strong noncovalent interactions. A more polar cyclic peptide might produce a much different DR-ECD spectrum, as in the case of gramicidin S (cyclo-VOLFPVOLFP), where product ion depletion was observed in some but not all of the secondary fragments (Figure $5 d$ ). The charged orthinine side-chain was probably involved in keeping two secondary fragments together for a short period of time once they were formed. In addition to the secondary backbone cleavage fragments, there were also peaks corresponding to additional loss of $\mathrm{CONH}$, which completely disappeared in the DR-

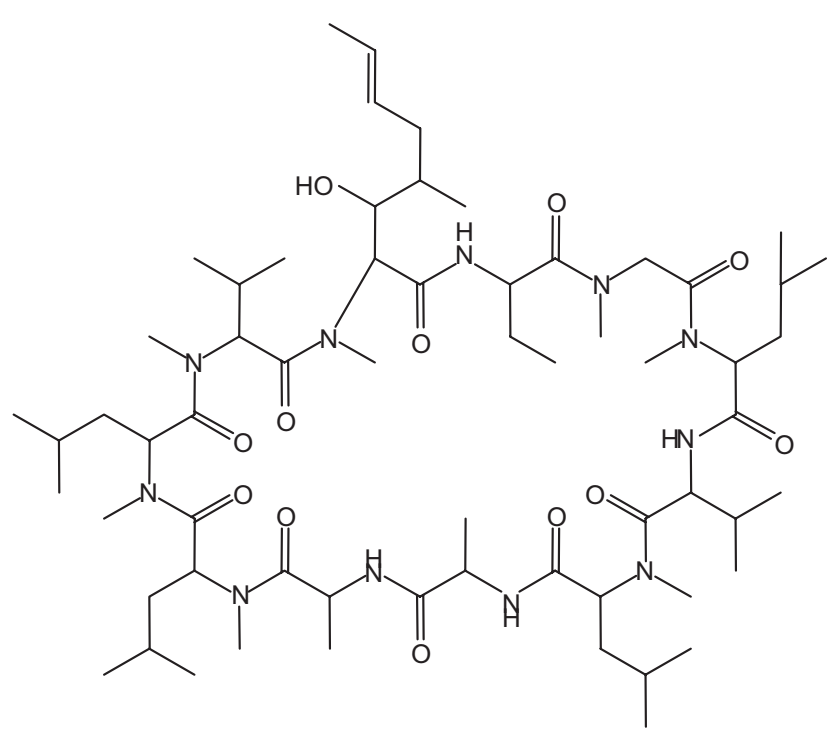

Scheme 1. Cyclosporin A structure. 


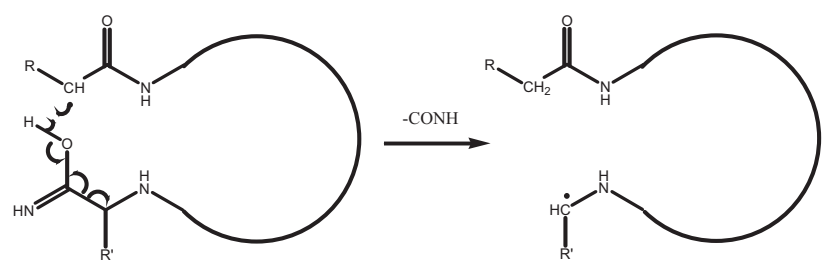

Scheme 2. A possible mechanism to explain the $-\mathrm{CONH}$ loss peaks in cyclic peptide ECD spectra.

ECD spectrum. The CONH loss might be the result of a radical rearrangement of the type shown in Scheme 2, which is typically slow due to a rather specific steric requirement.

So far, the resonance ejection has been applied only to the charge reduced molecular ions, but it can also be applied at other frequencies. To test the free radical cascade in linear peptide ECD, an on-resonant ejection waveform has been applied to eject various sized $\mathrm{c}$ and $\mathrm{z}$ ions of the BUSM 1 peptide. In light of what has been seen in the cyclic peptide DR-ECD experiment, it did not come as a big surprise that no change in smaller fragment ion abundance was observed (spectra not shown). The radical cascade might still be happening in these peptides, but since it is an intramolecular radical induced reaction, it is too fast to be captured by the DR-ECD experiment.

\section{DR-ECD of Multiply Charged Peptide Ions (Melittin)}

When we move on to larger peptides where the precursor ions can carry more than two positive charges, it is now possible to eject not only the singly-reduced molecular ion, but also the doubly-reduced ones. One particular interest for doing the latter is to evaluate the importance of secondary electron capture in ECD experiment. It has been reported that secondary electron capture was not important in Substance P ECD [22], however, a more highly charged peptide ion might display a different behavior. Since electron capture efficiency increases quadratically with the number of the charges [2], a highly charged charge reduced molecular ion is much more likely to undergo secondary electron capture than the singly-charged Substance P reduced ion. Figure 6 shows the ECD spectrum of the quadruply charged melittin (Figure 6a), as well as its DR-ECD spectra with resonant rejection of the singlyreduced molecular ion $[\mathrm{M}+4 \mathrm{H}]^{3+\cdot}(m / z \sim 949.6$, Figure $6 \mathrm{~b})$ and the doubly-reduced molecular ion $[\mathrm{M}+4 \mathrm{H}]^{2+. *}$ $(\mathrm{m} / \mathrm{z} \sim 1424.4$, Figure 6c). It is apparent from the spectra that secondary electron capture was readily present and accounted for the formation of a large percentage of many singly-charged fragment ions: $z_{5}, z_{6}, z_{11}, z_{12}, c_{12}$, $\mathrm{c}_{17}$, and $\mathrm{c}_{18}$, as the ejection of $[\mathrm{M}+4 \mathrm{H}]^{2+\cdot}$ resulted in a big drop in their peak intensities. Additional evidence pointing towards the origin of these ions from the doubly-reduced molecular ion existed in the distorted isotopic pattern (Figure 6, inset), where the isotopic distribution of the $\mathrm{z}_{11}^{+}$ion appeared to shift one $\mathrm{Da}$ higher. Several of the fragment ions appeared in more than one charge state, e.g., $\mathrm{z}_{12}, \mathrm{z}_{16}$, and the lower charge state fragment ion usually showed higher depletion upon resonance ejection of $[\mathrm{M}+4 \mathrm{H}]^{3+}$. As pointed out earlier, these lower charge state fragment ions arose from the $[\mathrm{M}+4 \mathrm{H}]^{2+.}$, which itself came from the

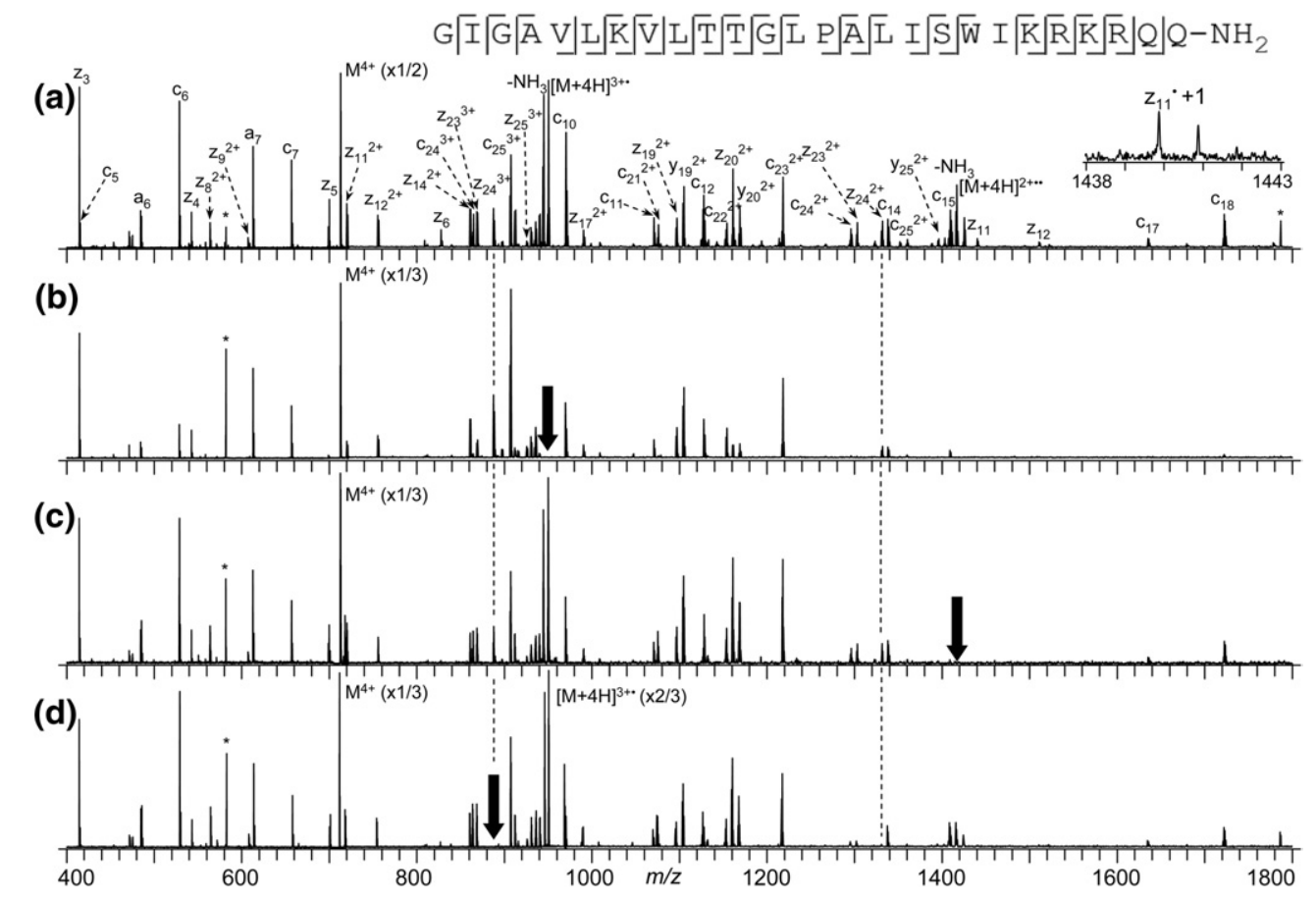

Figure 6. Melittin 4+ ECD spectrum (a) and its DR-ECD spectra with the resonant ejection of the [M $+4 \mathrm{H}]^{3+\cdot}$ ion $(\mathbf{b})$, the $[\mathrm{M}+4 \mathrm{H}]^{2+\cdot \cdot}$ ion $(\mathbf{c})$, and the $\mathrm{c}_{24}^{3+}$ ion $(\mathbf{d})$. 


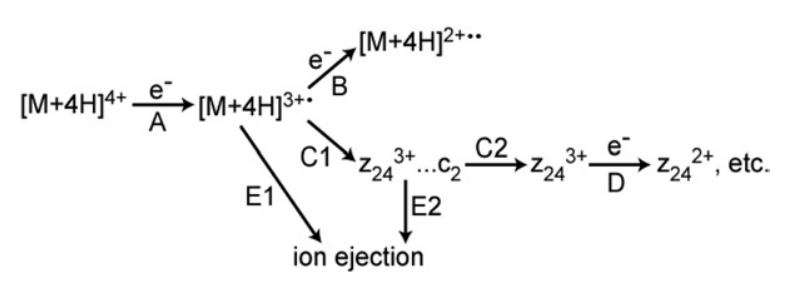

Scheme 3. Various processes that lead to the formation and consumption of the $z_{24}^{3+}$ ion in the ECD of the quadruply charged melittin ion.

secondary electron capture of $[\mathrm{M}+4 \mathrm{H}]^{3+}$. Unlike the fragment ions that require ejection of the $[\mathrm{M}+4 \mathrm{H}]^{3+}$. ion out of the cell to be completely absent in the DR-ECD spectrum, the $[\mathrm{M}+4 \mathrm{H}]^{2+.}$ ion (and the fragment ions from it) would disappear from the spectrum even if the $[\mathrm{M}+4 \mathrm{H}]^{3+\cdot}$ ion is ejected to just outside of the electron cloud, which has a much smaller diameter $(\sim 2.5 \mathrm{~mm})$ than the cell diameter $(\sim 75 \mathrm{~mm})$. Furthermore, as the $[\mathrm{M}+4 \mathrm{H}]^{3+}$ ion gets excited, its relative speed with respect to the electron increases and its electron capture efficiency drops, although this is probably not a big factor, as the ion velocity at the edge of the electron cloud is only a few percent of that of the $0.25 \mathrm{eV}$ electron.

While generally not observed in the DR-ECD spectra of the doubly-charged peptide ions, some fragment ions (all multiply charged ones) from the quadruply charged melittin ion showed increase in their abundance upon $[\mathrm{M}+4 \mathrm{H}]^{3+\cdot}$ ejection, e.g., $\mathrm{z}_{24}^{3+}$ in Figure 6. To explain this, it is necessary to understand the various processes that lead to the production and consumption of the $z_{24}^{3+}$ ion, as illustrated in Scheme 3. Process $A$ is the electron capture of the precursor ion, leading to the formation of the charge reduced $[\mathrm{M}+4 \mathrm{H}]^{3+\cdot}$ ion, which can subsequently produce the $z_{24}^{3+}$ ion via process $C$. A competing pathway to this $z_{24}^{3+}$ ion forming channel is the secondary electron capture of the $[\mathrm{M}+4 \mathrm{H}]^{3+\cdot}$ ion to produce the $[\mathrm{M}+4 \mathrm{H}]^{2+. .}$ ion (process $\mathrm{B}$ ), which cannot generate the $\mathrm{z}_{24}^{3+}$ ion. Process B can be blocked if the $[\mathrm{M}+4 \mathrm{H}]^{3+}$. ion is ejected (process $\mathrm{E}$ ) outside of the electron cloud as soon as it is formed. Any ejecting $[\mathrm{M}+4 \mathrm{H}]^{3+\cdot}$ ion (or the $\left[z_{24}^{3+} \ldots c_{2}\right]$ complex, as the two cannot be differentiated in this experiment) falling apart beyond the edge of the electron cloud but still within the boundary of the cell can generate a detectable $z_{24}^{3+}$ ion, which may not be present if the $[\mathrm{M}+4 \mathrm{H}]^{3+\cdot}$ ion is left in the center of the ICR cell (i.e., no resonant ejection) to undergo possible secondary electron capture. If the contribution from these extra $z_{24}^{3+}$ ions generated in the DR-ECD experiment outweighs the loss of ions and ion coherence due to the resonant ejection, a survival ratio greater than 1 may be expected.

Secondary electron capture can also take place in the $z_{24}^{3+}$ fragment ion itself to form the $z_{24}^{2+}$ ion (process D), resulting in the loss of the $z_{24}^{3+}$ ion population. Process D can only occur when the originally generated $z_{24}^{3+}$ ion stays inside the electron cloud, evident by the $z_{24}^{2+}$ ion abundance drop upon resonant ejection of the $z_{24}^{3+}$ ion during ECD (Figure 6d). In addition, several smaller fragment ions, e.g., $z_{23}^{2+}$, also showed appreciable drop in population upon the $z_{24}^{3+}$ ejection. These ions could be the secondary fragment ions from the free radical cascade of the $z_{24}^{3+}$ ion, but most likely, they were from ECD of the $z_{24}^{3+}$ ions, as none of them carried more than two charges. Ejection of the $[\mathrm{M}+4 \mathrm{H}]^{3+\cdot}$ ion outside of the electron cloud before fragment ion separation may generate detectable $z_{24}^{3+}$ ion that would otherwise (as in the normal ECD experiments) be subject to possible secondary electron capture. Once again, if the contribution from these "escaped" (from secondary electron capture) $z_{24}^{3+}$ ions outweighs the ion coherence loss due to resonant ejection, it is possible to observe an ion abundance increase in the DR-ECD experiment.

Secondary electron capture can occur in doublycharged peptide ions as well, although the resulting fragments cannot be detected in normal ECD experiment since they carry no charges. Since survival ratio is the ratio of the ion abundance with resonant ejection versus the normal ECD ion abundance, and secondary electron capture will affect the latter much more than the former, secondary electron capture in DR-ECD experiments often results in survival ratios $>1$. One such example is given in Figure $4 b$, where a few $c$ ions from the BUSM 1 peptide ECD showed increases in abundance upon resonant ejection. The increase, however, is not as pronounced as in the case of the multiply charged fragment ions, as expected from their drastic difference in electron capture efficiencies.

Quantitative assessment of the lifetime of the intermediates that produce highly charged fragment ions is complicated by the secondary electron capture of both the charge reduced $[\mathrm{M}+4 \mathrm{H}]^{3+\cdot}$ ion and the fragment ion itself, as well as the uncertainty on the ejection radius. A rough estimate requires knowledge of the relative rates of various processes in Scheme 3. Resonant ejection of the $[\mathrm{M}+4 \mathrm{H}]^{3+\cdot}$ ion eliminates reaction $\mathrm{B}$ in Scheme 3 and, thus, also eliminates all fragmentation channels derived from the $[\mathrm{M}+4 \mathrm{H}]^{2+\cdot}$ ion. Thus, in Scheme 3, any ion reaction that competes directly with formation of the $[\mathrm{M}+4 \mathrm{H}]^{2+\cdot \cdot}$ ion (such as reaction C1/C2) will be enhanced upon elimination of reaction B. The amount of the $[\mathrm{M}+4 \mathrm{H}]^{3+\cdot}$ ion that underwent secondary electron capture is the sum of the abundance of the $[\mathrm{M}+4 \mathrm{H}]^{2+\cdot \cdot}$ ion and everything derived from it, which should be the difference between Spectrum 6a and Spectrum 6c. Only a handful of ions showed significant drop in their peak intensities upon resonance ejection of the $[\mathrm{M}+4 \mathrm{H}]^{2+\cdot \cdot}$ ion, and they consisted of less than $10 \%$ of the total fragment ions generated by ECD of the molecular ion. Therefore, the maximum increase in survival ratio of any ion upon resonance ejection of the $[\mathrm{M}+4 \mathrm{H}]^{3+\cdot}$ ion due to its escape from secondary electron capture cannot exceed 1.1. The importance of the secondary electron capture of the $z_{24}^{3+}$ fragment ion itself, on the other hand, can be quite significant. In the normal ECD spectrum of the quadruply charged melittin ion, the $z_{24}^{2+}$ ion had an 
abundance about the same as that of the $z_{24}^{3+}$ ion (after correction for the number of charges), but almost disappeared when the $z_{24}^{3+}$ ion was ejected $\left(40 V_{p-p}\right)$ during ECD (Figure 6d). Even if all other fragment ions that dropped in intensity were the result of the free radical cascade dissociation of the $z_{24}^{3+}$ ion, the elimination of secondary electron capture by resonance ejection could account for an $\sim 2$ fold increase in abundance. The fact that the survival ratio of $z_{24}^{3+}$ on ejection of the $[\mathrm{M}+4 \mathrm{H}]^{3+\cdot}$ ion is close to 2 means that most escaped $[\mathrm{M}+4 \mathrm{H}]^{3+\cdot}$ ions fell apart shortly after they were ejected outside of the electron cloud, but long before they hit the cell wall, otherwise the coherence loss will decrease that value significantly. At $40 \mathrm{~V}_{p-p}$ ejection voltage, complete ion ejection takes around $1 \mathrm{~ms}$, and partial ejection (outside of the electron cloud) takes about $35 \mu \mathrm{s}$, but could be up to two times longer due to the imperfect alignment of the electron and the ion beam. The intermediate that produced fragment ions with survival ratios greater than one probably has a lifetime closer to what it takes to eject the ion outside of the electron cloud than to the ms lifetime of those that showed significant drops in DR-ECD. It is thus reasonable to believe that fragment ion peaks that increased in intensity upon resonant ejection originated from intermediates that have somewhat weak noncovalent interactions, much like those fragment ions that showed no change in abundance in DR-ECD experiments.

\section{Correlation of Fragment Ion Survival Ratios with Gas Phase Conformation}

The survival ratios of various $\mathrm{c} / \mathrm{z}$ ions from melittin $4+$ DR-ECD are listed in Table 1, but it is not immediately clear how to construct its gas-phase conformation from this information. In organic solvents, melittin exists in an $\alpha$-helical form with two $\alpha$-helices, one at each end, connected by a nonhelical hinge around Pro14 [40]. In their mass-analyzed ion kinetic energy (MIKE) spectrometry studies, Kaltashov and Fenselau have shown that triply charged melittin ion probably retains this helical structure in the gas-phase [41]. The conclusion was drawn based on a single parameter of intercharge distance between Lys7 and Arg22/24, the proposed charged sites, as determined by kinetic energy release accompanying fragmentation. Their molecular dynamics calculations supported the retention of helical structure in the gas phase, and it also pointed out that the proton carrying side-chains were involved in intramolecular hydrogen binding to stabilize the charges in the absence of solvent molecules.

The gas-phase conformation of the quadruply charged melittin ion is less well known. H/D exchange studies by Hakansson's group found the amide hydrogens of Leu13 and Ala15 near the unstructured kink to be fast exchangers, while those of the Leu16 and Lys23 to be slow ones, possibly due to their involvement in the helix formation [42]. The observation of $c_{5}$ and $c_{6}$
Table 1. Survival ratios for melittin $4+$ DR-ECD on $[\mathrm{M}+4 \mathrm{H}]^{3+\cdot}$ ion

\begin{tabular}{|c|c|c|c|c|c|c|c|c|}
\hline & C & $\mathrm{C}^{+}$ & $\mathrm{C}^{2+}$ & $\mathrm{C}^{3+}$ & Z & $\mathrm{Z}^{+}$ & $\mathrm{Z}^{2+}$ & $\mathrm{Z}^{3+}$ \\
\hline $\mathrm{G}$ & 1 & & & & 26 & & & \\
\hline I & 2 & & & & 25 & & & 1.95 \\
\hline $\mathrm{G}$ & 3 & & & & 24 & & 0.52 & 1.85 \\
\hline A & 4 & & & & 23 & & 0.10 & 0.50 \\
\hline V & 5 & 0.34 & & & 22 & & & \\
\hline L & 6 & 0.27 & & & 21 & & & \\
\hline K & 7 & 0.70 & & & 20 & & 0.20 & \\
\hline V & 8 & 0.71 & & & 19 & & 1.20 & \\
\hline L & 9 & 0.61 & & & 18 & & & \\
\hline $\mathrm{T}$ & 10 & 0.56 & & & 17 & & 0.87 & \\
\hline $\mathrm{T}$ & 11 & 0.72 & & & 16 & 0 & $0.18^{a}$ & \\
\hline $\mathrm{G}$ & 12 & 0.86 & & & 15 & & 1.32 & \\
\hline $\mathrm{L}$ & 13 & & & & 14 & & 1.17 & \\
\hline$P$ & 14 & 0.46 & & & 13 & & & \\
\hline A & 15 & 0.24 & & & 12 & 0 & 0.81 & \\
\hline L & 16 & & & & 11 & 0 & 0.45 & \\
\hline I & 17 & 0 & & & 10 & & & \\
\hline$S$ & 18 & 0.15 & & & 9 & 0 & 0 & \\
\hline W & 19 & & & & 8 & 0 & 0 & \\
\hline I & 20 & & & & 7 & & & \\
\hline $\mathrm{K}$ & 21 & & 0.14 & & 6 & 0 & & \\
\hline $\mathrm{R}$ & 22 & & 1.40 & & 5 & 0.21 & & \\
\hline K & 23 & & 1.31 & & 4 & 0.92 & & \\
\hline $\mathrm{R}$ & 24 & & 0.16 & 0.17 & 3 & 0.91 & & \\
\hline $\mathrm{Q}$ & 25 & & 0.46 & 2.10 & 2 & & & \\
\hline $\mathrm{Q}-\mathrm{NH} 2$ & 26 & & & & 1 & & & \\
\hline
\end{tabular}

${ }^{a}$ Marks the fragment ion whose $\mathrm{m} / \mathrm{z}$ is very close to that of the ejected ion which renders its survival ratio unreliable.

ions in the ECD spectrum of the melittin 4+ ion (Figure 6) suggested that the fourth charge resides on the $\mathrm{N}$-terminal glycine, as would be expected from minimization of columbic repulsion. Hudgins and Jarrold found that the addition of $C$-terminal charge stabilizes the helical structure by the interaction of the charge with the helix dipole, but the $\mathrm{N}$-terminal charge has no such effect [43]. If the helix near the $N$-terminus is disrupted somehow by the addition of a charge, it would mean that the $\mathrm{N}$-terminal $-\mathrm{NH}_{2}$ group and the charged Lys7 side-chain have more flexibility to interact with the $C$-terminal glutamine side-chains.

Ions showing the biggest drop in abundance upon resonance ejection of the $[\mathrm{M}+4 \mathrm{H}]^{3+\cdot}$ ion are mostly singly-charged $\mathrm{z}$ ions that contained both arginines. Previous studies found that charges attached to the more basic sites are less likely to react with an electron [44], neutralization of at least one arginine side-chain in these fragment ions implies that they might result from the double electron capture of the precursor ion. Further support of this hypothesis comes from the fact that their complementary $\mathrm{c}^{2+}$ ions are missing from the spectrum. Incidentally, these ions also have the smallest survival ratio upon resonance ejection of the $[\mathrm{M}+$ $4 \mathrm{H}]^{2+\cdot}$ ion, which itself is highly susceptible to the resonance ejection of the $[\mathrm{M}+4 \mathrm{H}]^{3+\cdot}$ ion (out of the electron cloud), explaining their absence (or nearly so) in the DR-ECD spectrum.

For singly-charged $c$ ions, the survival ratios of the 
smallest two observed ( $c_{5}$ and $c_{6}$ ions) are among the lowest, those of the intermediate ones are moderate, between 0.5 and 1 , and the value falls again for ions resulting from cleavage past Pro13. As demonstrated by Williams and coworkers, backbone cleavages are most likely occurring near the charge sites [45]. For $c_{5}$ and $c_{6}$ ions, the most likely charge neutralization that induced their formation is the side-chain of Lys7. Since these two ions retained the charge on $\mathrm{N}$-terminal glycine, which is quite flexible to interact freely with the $\mathrm{C}$-terminal polar groups, their separation from their complementary zions were hindered. The addition of another possible charge site in Lys7 in $c_{7}$ through $c_{12}$ ions means that the likely neutralization site is now the $N$-terminal glycine, and the Lys7 side-chain is the charge carrier. The necessity of hydrogen bonds between $N$-terminal $-\mathrm{NH}_{3}^{+}$group and the carbonyl next to the cleavage site locked the lysine side-chain in a relatively tight position, and since the stable alpha helix near $C$-terminus affords little flexibility, the interaction between Lys7 side-chain and C-terminal polar groups is limited, resulting in the increased survival ratio. As the cleavage site gets past the kink region near Pro14, the hydrogen bond between the $\mathrm{N}$-terminal glycine and the carbonyl bends the $\mathrm{N}$-terminal helix (and the charged Lys7 side-chain) further down towards the $C$-terminal helix, making the interaction between Lys7 side-chain and Gln $25 / 26$ possible, and smaller survival ratio as a direct result.

A similar trend is also observed for the complementary $z_{n}^{2+}$ ion series, where cleavage before Lys7 and after Pro14 resulted in ions with smallest survival ratio, and those in the middle resulted in the largest one. Some $\mathrm{z}_{\mathrm{n}}^{2+}$ ions have survival ratios larger than one, because of the secondary electron capture of the fragment ions as stated earlier, which is more likely to happen in higher charge state ions.

The doubly-charged $\mathrm{c}$ ions, $\mathrm{c}_{24}^{2+}$ and $\mathrm{c}_{25}^{2+}$, have small survival values because they were likely the product of double electron capture, since they contain all four possible charged sites. Formation of the $\mathrm{c}_{22}^{2+}$ and $\mathrm{c}_{23}^{2+}$ ions resulted from the neutralization of $\mathrm{N}$-terminal glycine $-\mathrm{NH}_{3}^{+}$group, which was probably hydrogen bonded to the carbonyl of the Lys21 and Arg22, respectively, preventing Lys7 side-chain from binding to the $C$ terminal glutamine side-chains, thus resulting in relatively large survival ratios. The $c_{21}^{2+}$ ion formation required Arg22 side-chain to hydrogen bond to Lys21 carbonyl, since it retained charge on both Gly1 and Lys7, allowing these two charged sites to interact freely with C-terminal glutamine side-chains, yielding a significant drop in abundance upon resonance ejection of the $[\mathrm{M}+4 \mathrm{H}]^{3+\cdot}$ ion.

Finally, many triply charged fragment ions have survival ratios exceeding the theoretical maximum of one substantially. This is because triply charged fragment ions are even more vulnerable to secondary electron capture than the doubly-charged ones. The stark difference in survival ratio between the $\mathrm{c}_{24}^{3+}$ and
$\mathrm{C}_{25}^{3+}$ ion might arise from the availability of the Cterminal glutamine side-chains to interact with the Lys7 side-chain. The necessity of Gly1 $-\mathrm{NH}_{3}^{+}$group to hydrogen bond to Gln25 carbonyl to induce the cleavage that generates $\mathrm{c}_{25}^{3+}$ ions is likely to render the $G \ln 26$ side-chain inaccessible for Lys7, which would not pose the same constraint when the hydrogen bond was one residue further away in the case of $\mathrm{c}_{24}^{3+}$.

It is important to note that gas-phase peptide ions likely exist in multiple conformations, which also accounts for some of the survival ratio variations. The charge reduced molecular ion(s) are expected to have different conformations from the hydrogen loss products, due to the presence of additional hypervalent sites. These conformations currently can only be probed by spectroscopic $[32,46]$ or computational methods [47, 48 , which are beyond the scope of this paper.

\section{Conclusions}

The fragment ion peak intensities in ECD of protein ions are often affected by their gas-phase conformations. Peptide ions usually have weaker intra-molecular bonds whose effects are often too small to significantly alter the relative abundances of the ECD fragment ions. Because of the fast ejection timescale, DR-ECD can be used instead to detect the existence of these short-lived intermediates that were held together by weak noncovalent interactions by observing the change of fragment ion peak intensities upon resonant ejection of the charge reduced molecular species. The survival ratio of a fragment ion directly correlates to the lifetime of the intermediate that generates it, which depends mostly on the secondary and tertiary structures of the precursor ion. Gas-phase conformations of several peptide ions were proposed based on the survival ratios of fragment ions in DR-ECD experiments. The validity of this approach was supported by the dissimilar behavior of a set of peptides with similar sequence, and in one case, by comparison with the established structure in the literature.

As shown in the example of fibrinopeptide B, the lifetime of these radical intermediates can sometimes exceed a ms. Multiple radical rearrangements can happen in this time, allowing processes such as free radical cascade and intramolecular hydrogen transfer to occur. The odd electron c. fragment ions in the BUSM 1 peptide ECD appeared to have much smaller survival ratios than the corresponding even electron $\mathrm{c}$ ions, marking their different origins, with the former likely being the result of intramolecular hydrogen transfer. Survival ratios larger than one have been observed for some multiply charged fragment ions in the DR-ECD of quadruply charged melittin ion. This implies that secondary electron capture can be quite significant in the ECD of highly charged peptide ions.

Finally, as demonstrated in this paper, DR-ECD experiments can be a handy tool in the study of ECD 
mechanism as well as gas-phase conformations of peptide and protein ions.

\section{Acknowledgments}

The authors thank R. A. Zubarev for helpful discussions. This work has been supported by NIH/NCRR P41RR10888, NIH/ NHLBI N01HV28178, MDS Sciex, and the American Chemical Society Petroleum Research Fund.

\section{References}

1. Zubarev, R. A.; Kelleher, N. L.; McLafferty, F. W. Electron Capture Dissociation of Multiply Charged Protein Cations. A Nonergodic Process. J. Am. Chem. Soc. 1998, 120, 3265-3266.

2. Zubarev, R. A.; Horn, D. M.; Fridriksson, E. K.; Kelleher, N. L.; Kruger, N. A.; Lewis, M. A.; Carpenter, B. K.; McLafferty, F. W. Electron Capture Dissociation for Structural Characterization of Multiply Charged Protein Cations. Anal. Chem. 2000, 72, 563-573.

3. McLafferty, F. W.; Horn, D. M.; Breuker, K.; Ge, Y.; Lewis, M. A.; Cerda, B.; Zubarev, R. A.; Carpenter, B. K. Electron Capture Dissociation of Gaseous Multiply Charged Ions by Fourier-Transform Ion Cyclotron Resonance. I. Am. Soc. Mass Spectrom. 2001, 12, 245-249.

4. Cooper, H. J.; Hakansson, K.; Marshall, A. G. The Role of Electron Capture Dissociation in Biomolecular Analysis. Mass Spectrom. Rev. 2005, 24, 201-222.

5. Leymarie, N.; Costello, C. E.; O'Connor, P. B. Electron Capture Dissociation Initiates a Free Radical Reaction Cascade. J. Am. Chem. Soc. 2003, 125, 8949-8958.

6. Kelleher, R. L.; Zubarev, R. A.; Bush, K.; Furie, B.; Furie, B. C.; McLafferty, F. W.; Walsh, C. T. Localization of Labile Posttranslational Modifications by Electron Capture Dissociation: The Case of $\gamma$-Carboxyglutamic Acid. Anal. Chem. 1999, 71, 4250-4253.

7. Mirgorodskaya, E.; Roepstorff, P.; Zubarev, R. A. Localization of O-Glycosylation Sites in Peptides by Electron Capture Dissociation in a Fourier Transform Mass Spectrometer. Anal. Chem. 1999, 71, 4431-4436.

8. Stensballe, A.; Jensen, O. N.; Olsen, J. V.; Haselmann, K. F.; Zubarev, R. A. Electron Capture Dissociation of Singly and Multiply Phosphorylated Peptides. Rapid Commun. Mass Spectrom. 2000, 14, 1793-1800.

9. Shi, S. D. H.; Hemling, M. E.; Carr, S. A.; Horn, D. M.; Lindh, I. McLafferty, F. W. Phosphopeptide/Phosphoprotein Mapping by Electron Capture Dissociation Mass Spectrometry. Anal. Chem. 2001, 73, $19-22$.

10. Zhao, C.; Sethuraman, M.; Clavreul, N.; Kaur, P.; Cohen, R. A.; O'Connor, P. B. A Detailed Map of Oxidative Post-Translational Modifications of Human p21ras Using Fourier Transform Mass Spectrometry. Anal. Chem. 2006, 78, 5134-5142.

11. Syrstad, E. A.; Turecek, F. Toward a General Mechanism of Electron Capture Dissociation. J. Am. Soc. Mass Spectrom. 2005, 16, 208-224.

12. O'Connor, P. B.; Lin, C.; Cournoyer, J. J.; Pittman, J. L.; Belyayev, M.; Budnik, B. A. Long-Lived Electron Capture Dissociation Product Ions Experience Radical Migration via Hydrogen Abstraction. J. Am. Soc. Mass Spectrom. 2006, 17, 576-585.

13. Zubarev, R. A. Reactions of Polypeptide Ions with Electrons in the Gas Phase. Mass Spectrom. Rev. 2003, 22, 57-77.

14. Asam, M. R.; Glish, G. L. Determination of the Dissociation Kinetics of a Transient Intermediate. I. Am. Soc. Mass Spectrom. 1999, 10, 119-125.

15. Anders, L. R.; Beauchamp, J. L.; Dunbar, R. C.; Baldeschwieler, J. D. Ion-Cyclotron Double Resonance. J. Chem. Phys. 1966, 45, 1062-1063.

16. Comisarow, M. B.; Marshall, A. G. Fourier Transform Ion Cyclotron Resonance Spectroscopy. Chem. Phys. Lett. 1974, 25, 282-283.

17. Marshall, A. G.; Hendrickson, C. L.; Jackson, G. S. Fourier Transform Ion Cyclotron Resonance Mass Spectrometry: A primer. Mass Spectrom. Rev. 1998, 17, 1-35.

18. Asam, M. R.; Glish, G. L. Tandem Mass Spectrometry of Alkali Cationized Polysaccharides in a Quadrupole Ion Trap. J. Am. Soc. Mass Spectrom. 1997, 8, 987-995.

19. Comisarow, M. B.; Grassi, V.; Parisod, G. Fourier Transform Ion Cyclotron Double Resonance. Chem. Phys. Lett. 1978, 57, 413-416.

20. Jurchen, J. C.; Garcia, D. E.; Williams, E. R. Gas-Phase Dissociation Pathways of Multiply Charged Peptide Clusters. J. Am. Soc. Mass Spectrom. 2003, 14, 1373-1386.

21. Anicich, V. G.; Sen, A. D.; Huntress, W. T.; McEwan, M. J. Lifetime Measurement of a Collision Complex Using Ion-Cyclotron DoubleResonance $\left(\mathrm{H}_{2} \mathrm{C}_{6} \mathrm{~N}_{2}\right)+$. J. Chem. Phys. 1991, 94, 4189-4191.

22. Budnik, B. A.; Nielsen, M. L.; Olsen, J. V.; Haselmann, K. F.; Horth, P.; Haehnel, W.; Zubarev, R. A. Can Relative Cleavage Frequencies in Peptides Provide Additional Sequence Information? Int. J. Mass Spectrom. 2002, 219, 283-294.

23. Suckau, D.; Shi, Y.; Beu, S. C.; Senko, M. W.; Quinn, J. P.; Wampler, F. M.; McLafferty, F. W. Coexisting Stable Conformations of Gaseous Protein Ions. Proc. Natl. Acad. Sci. U.S.A. 1993, 90, 790-793.
24. Wood, T. D.; Chorush, R. A.; Wampler, F. M.; Little, D. P.; O'Connor, P. B.; McLafferty, F. W. Gas-Phase Folding and Unfolding of Cytochrome-c Cations. Proc. Natl. Acad. Sci. U.S.A. 1995, 92, 2451-2454.

25. Freitas, M. A.; Hendrickson, C. L.; Emmett, M. R.; Marshall, A. G. Gas-Phase Bovine Ubiquitin Cation Conformations Resolved by GasPhase Hydrogen/Deuterium Exchange Rate and Extent. Int. J. Mass Spectrom. 1999, 187, 565-575.

26. Smith, D. L.; Deng, Y. Z.; Zhang, Z. Q. Probing the Noncovalent Structure of Proteins by Amide Hydrogen Exchange and Mass Spectrometry. J. Mass Spectrom. 1997, 32, 135-146.

27. Valentine, S. J.; Counterman, A. E.; Clemmer, D. E. Conformer-Dependent Proton-Transfer Reactions of Ubiquitin Ions. J. Am. Soc. Mass Spectrom. 1997, 8, 954-961.

28. Counterman, A. E.; Clemmer, D. E. Large Anhydrous Polyalanine Ions: Evidence for Extended Helices and Onset of a More Compact State. J. Am. Chem. Soc. 2001, 123, 1490-1498.

29. Wyttenbach, T.; Kemper, P. R.; Bowers, M. T. Design of a New Electrospray Ion Mobility Mass Spectrometer. Int. J. Mass Spectrom. 2001, 212, 13-23.

30. Kinnear, B. S.; Hartings, M. R.; Jarrold, M. F. The Energy Landscape of Unsolvated Peptides: Helix Formation and Cold Denaturation in AcA(4)G(7)A4+H+. J. Am. Chem. Soc. 2002, 124, 4422-4431.

31. Oh, H.; Breuker, K.; Sze, S. K.; Ge, Y.; Carpenter, B. K.; McLafferty, F. W. Secondary and Tertiary Structures of Gaseous Protein Ions Characterized by Electron Capture Dissociation Mass Spectrometry and Photofragment Spectroscopy. Proc. Natl. Acad. Sci. U.S.A. 2002, 99, 1586315868

32. Oomens, J.; Polfer, N.; Moore, D. T.; van der Meer, L.; Marshall, A. G.; Eyler, J. R.; Meijer, G.; von Helden, G. Charge-State Resolved MidInfrared Spectroscopy of a Gas-Phase Protein. Phys. Chem. Chem. Phys. 2005, 7, 1345-1348.

33. Horn, D. M.; Breuker, K.; Frank, A. J.; McLafferty, F. W. Kinetic Intermediates in the Folding of Gaseous Protein Ions Characterized by Electron Capture Dissociation Mass Spectrometry. J. Am. Chem. Soc. 2001, 123, 9792-9799.

34. Breuker, K.; Oh, H. B.; Horn, D. M.; Cerda, B. A.; McLafferty, F. W. Detailed Unfolding and Folding of Gaseous Ubiquitin Ions Characterized by Electron Capture Dissociation. J. Am. Chem. Soc. 2002, 124, 6407-6420.

35. Breuker, K.; McLafferty, F. W. Native Electron Capture Dissociation for the Structural Characterization of Noncovalent Interactions in Native Cytochrome c. Angew. Chem. Int. Ed. 2003, 42, 4900-4904.

36. Mihalca, R.; Kleinnijenhuis, A. J.; McDonnell, L. A.; Heck, A. J. R.; Heeren, R. M. A. Electron Capture Dissociation at Low Temperatures Reveals Selective Dissociations. J. Am. Soc. Mass Spectrom. 2004, 15, $1869-1873$

37. Jebanathirajah, J. A.; Pittman, J. L.; Thomson, B. A.; Budnik, B. A.; Kaur, P.; Rape, M.; Kirschner, M.; Costello, C. E.; O'Connor, P. B. Characterization of a New qOq-FTICR Mass Spectrometer for Post-Translational Modification Analysis and Top-Down Tandem Mass Spectrometry of Whole Proteins. J. Am. Soc. Mass Spectrom. 2005, 16, 1985-1999.

38. O'Connor, P. B.; Pittman, J. L.; Thomson, B. A.; Budnik, B. A.; Cournoyer, J. C.; Jebanathirajah, J.; Lin, C.; Moyer, S.; Zhao, C. A New Hybrid Electrospray Fourier Transform Mass Spectrometer: Design and Performance Characteristics. Rapid Commun. Mass Spectrom. 2006, 20, $259-266$.

39. Newcomb, M. Competition Methods and Scales for Alkyl Radical Reaction-Kinetics. Tetrahedron 1993, 49, 1151-1176.

40. Bazzo, R.; Tappin, M. J.; Pastore, A.; Harvey, T. S.; Carver, J. A.; Campbell, I. D. The Structure of Melittin-a H-1-NMR Study in Methanol. Eur. J. Biochem. 1998, 173, 139-146.

41. Kaltashov, I. A.; Fenselau, C. Stability of Secondary Structural Elements in a Solvent-Free Environment: The $\alpha$ Helix. Proteins: Struct. Funct. Genet. 1997, 27, 165-170.

42. Kweon, H. K.; Hakansson, K. Site-Specific Amide Hydrogen Exchange in Melittin Probed by Electron Capture Dissociation Fourier Transform Ion Cyclotron Resonance Mass Spectrometry. Analyst 2006, 131, 275280 .

43. Hudgins, R. R.; Jarrold, M. F. Helix Formation in Unsolvated AlanineBased Peptides: Helical Monomers and Helical Dimers. J. Am. Chem. Soc. 1999, 121, 3494-3501.

44. Zubarev, R. A.; Haselmann, K. F.; Budnik, B.; Kjeldsen, F.; Jensen, F. Towards an Understanding of the Mechanism of Electron-Capture Dissociation: A Historical Perspective and Modern Ideas. Eur. J. Mass Spectrom. 2002, 8, 337-349.

45. Iavarone, A. T.; Paech, K.; Williams, E. R. Effects of Charge State and Cationizing Agent on the Electron Capture Dissociation of a Peptide. Anal. Chem. 2004, 76, 2231-2238.

46. Oh, H. B ; Lin, C.; Hwang, H. Y. Zhai, H. L.; Breuker, K. Zabrouskov, V.; Carpenter, B. K.; McLafferty, F. W. Infrared Photodissociation Spectroscopy of Electrosprayed Ions in a Fourier Transform Mass Spectrometer. J. Am. Chem. Soc. 2005, 127, 4076-4083.

47. Syrstad, E. A.; Stephens, D. D.; Turecek, F. Hydrogen Atom Adducts to the Amide Bond. Generation and Energetics of Amide Radicals in the Gas Phase. J. Phys. Chem. A 2003, 107, 115-126.

48. Patriksson, A.; Adams, C.; Kjeldsen, F.; Raber, J.; van der Spoel, D.; Zubarev, R. A. Prediction of $\mathrm{N}-\mathrm{C} \alpha$ Bond Cleavage Frequencies in Electron Capture Dissociation of Trp-Cage Dications by Force-Field Molecular Dynamics Simulations. Int. J. Mass Spectrom. 2006, 248, 124-135. 